九州大学学術情報リポジトリ

Kyushu University Institutional Repository

\title{
A System-Level Energy Minimization Approach Using Datapath Width Optimization
}

Cao, Yun

Department of Computer Science and Communication Engineering, Kyushu University

Yasuura, Hiroto

Department of Computer Science and Communication Engineering, Kyushu University

https://doi.org/10.15017/3433

出版情報: International Symposium on Low Power Electronics and Design (ISLPED’01)，pp. 231-236， 2001-08. Association for Computing Machinery

バージョン：

権利関係 : 


\title{
A System-level Energy Minimization Approach Using Datapath Width Optimization
}

\author{
Yun Cao Hiroto Yasuura \\ Department of Computer Science and Communication Engineering \\ Kyushu University \\ 6-1 Kasuga-koen, Kasuga-shi, Fukuoka 816-8580, Japan \\ $\{$ cao,yasuura\}@c.csce.kyushu-u.ac.jp
}

\begin{abstract}
This paper presents a novel system-level approach that minimizes the energy consumption of embedded core-based systems through datapath width optimization. It is based on the idea of minimizing energy consumed by redundant bits, which are unused during execution of programs by means of optimizing the datapath width of processors. To minimize the redundant bits of variables in a given application program, the effective size of each variable is determined by variable size analysis, and Valen-C language is used to preserve the precision of computation. Analysis results of variables show that there are average $39 \%$ redundant bits in the $\mathrm{C}$ source program of MPEG-2 video decoder. In our experiments for several embedded applications, energy savings without performance penalty are reported range from about $10.8 \%$ to $48.3 \%$.
\end{abstract}

\section{Keywords}

System-level energy minimization, variable size analysis, datapath optimization

\section{INTRODUCTION}

Minimizing power consumption of embedded systems is a crucial task. Battery-operated portable systems demand tight constraints on energy consumption. Better low-power circuit design techniques and advances in battery technology have helped to increase battery lifetime. On the other hand, managing power dissipation at higher design levels can considerably reduce energy consumption, and thus increase battery lifetime. Energy consumption at all design levels should be considered to reduce that of the whole embedded system.

We have developed a design platform, which consists of Valen-C retargetable compiler [2], soft-core processor (BungDLX) [1, 3] and a cycle-based simulator [4]. We also have done some researches on reduction of area and cost for em-

Permission to make digital or hard copies of all or part of this work for personal or classroom use is granted without fee provided that copies are not made or distributed for profit or commercial advantage and that copies bear this notice and the full citation on the first page. To copy otherwise, to republish, to post on servers or to redistribute to lists, requires prior specific permission and/or a fee.

ISLPED'01, August 6-7, 2001, Huntington Beach, California, USA

Copyright 2001 ACM 1-58113-371-5/01/0008 ...\$5.00. bedded core-based systems $[5,6]$. In this paper, we focus on minimizing energy dissipation and present a system-level approach for embedded core-based systems, which minimizes energy consumption of the whole system while providing adequate performance level. In the initial design phase of our approach, we design a system with a soft-core processor, data RAMs, instruction ROMs and logic circuits. Then we analyze the effective bit width of each variable of a given application program. After that, using the results of analysis, we rewrite the application program in Valen-C language [2], in which we specify the word length of each variable satisfying accurate computation to reduce energy consumed by redundant bits in the application program. After verifying the functionality of the initial design, we modify several design parameters of the soft-core processor, including the datapath width, the number of registers and the instruction set. We can tune up the soft-core processor to minimize the energy consumption while satisfying the system performance constraints. To get first-cut estimates of energy consumption early in the design, a few component-based power estimation models was also developed, total energy is obtained by summing over all components of the system.

This paper is structured as follows: the next Section 2 gives an overview of related work. Section 3 describes our energy minimization approach by datapath width optimization. Section 4 presents our energy estimation models. Experiments and results are shown in section 5. Finally, Section 6 concludes our work.

\section{RELATED WORK}

Hardware and software techniques to reduce energy consumption have become an essential part of current system designs. Extensive researches on power optimization from circuit level to system level have been conducted in these recent years. Such techniques have particularly targeted the memory system due to the prevalent use of data-dominated signal and video applications, such as [11], which focus on exploiting cache to reduce power consumption. The work [8] presented an architecture-oriented power minimization approach. A power and performance simulation tool that can be used to do architecture-level optimizations has been introduced by Sato et al. [9]. The approach [10] uses a multiple-voltage power supply to minimize system-power consumption. A framework for describing the power behavior of system-level designs was proposed by [7]. The paper [12] proposed a low power hardware/software partitioning approach using a high utilization rate of the involved resources. 


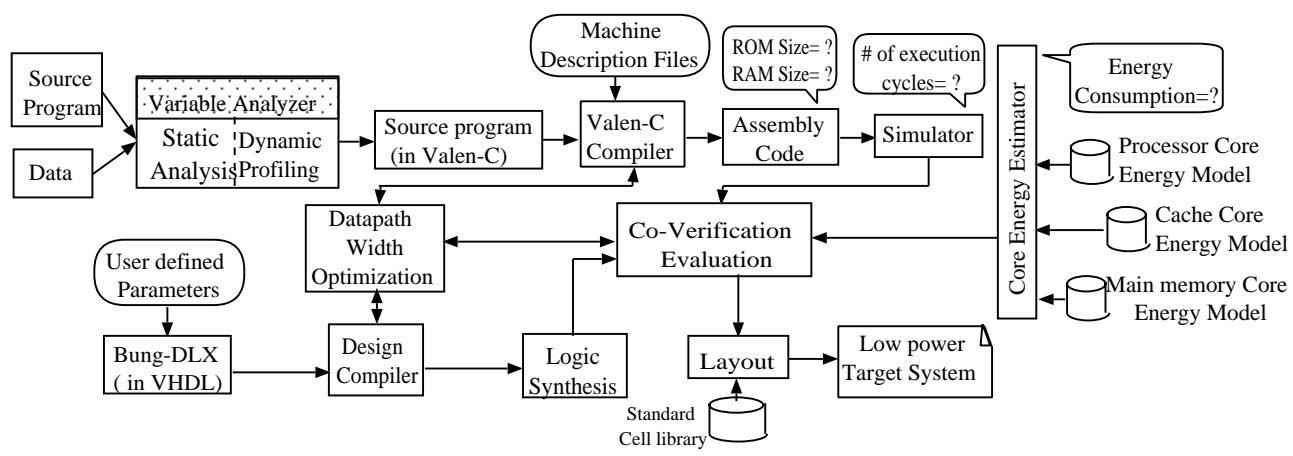

Figure 1: An energy minimization flow using datapath width optimization

As far as we know, this paper first present a system-level energy minimization approach in which designers can control the width of datapath freely. The energy consumption of the whole system is drastically reduced without decline of performance by optimizing the datapath width.

\section{AN ENERGY MINIMIZATION APPROACH}

In the design of consumer electronic systems, designers have to manage rapid increase of complexity of a target system with requirments on high-performance and low-energy comsumption under the tight constrait of short design time. Therefore, core-based solutions are proposed for embedded system design. Our approach gives designers a freedom to determine the datapath width of soft-core processor, because the datapath width of a processor has great impacts not only on power consumption and performance of the processor but also on those of memories. Optimizing datapath width for each given application is an effective approach to minimize energy consumption of the whole embedded systems. The energy minimization problem is stated as:

$$
\begin{array}{ll}
\text { minimize } & \text { Energy }(w) \\
\text { subject to } & \text { Cycle }(w) \leq C_{\text {cst }} \\
& \text { Area }(w) \leq A_{\text {cst }}
\end{array}
$$

Where Energy $(w)$, Cycle $(w)$ and Area $(w)$ are functions of the datapath width $w, C_{c s t}$ and $A_{c s t}$ are the constraints on the execution cycle and area respectively. This is a nonlinear optimization problem. The overview of our energy minimization algorithm is described in Figure2.

Figure1 shows our proposed approach, which consists of the following phases:

- Phase 1: The source program of the target application, which was originally written in $\mathrm{C}$ or other language, is rewritten in Valen-C language, after the bit width of each variable is analyzed. For instance, if the variable $x$ requires at most 11 bits, the programmer can write int11 $x$; in the variable declaration of Valen-C program.

- Phase 2: Bung-DLX is customized to different softcore processors by choosing different design parameters, such as the datapath width and the address size of the data memory.

- Phase 3: The Valen-C source program of an application is compiled for the customized soft-core processors. Retargetable Valen-C compiler generates the assembly code from the source program. As a result, different embedded systems are generated based on different customized processors and assembly codes. At this phase, the size of both the data memory and the instruction memory of each system are estimated.
- Phase 4: The systems generated at phase 3 are evaluated. Execution cycles, memory size and energy consumption are estimated. The impact of the design parameters on the energy consumption and on the system performance is evaluated, the embedded system of the minimal energy consumption, which satisfies the design constraints, is chosen among those systems.

\subsection{Variable Size Analysis}

In order to optimize datapath width, the effective size of each variable in an application program needs to be analyzed. This section explains our methods to analyze effective sizes of variables in $\mathrm{C}$ programs. In this paper, we define effective size as the smallest size which can hold both maximum and minimum values of a variable. In many cases, some bits of a variable are never used during execution of a program. If a variable $x$ of unsigned integer type whose value is in $[0,2000]$, i.e., between 0 and 2000 , then the number of necessary bits of $x$ is 11 , because the 11-bit size is large enough to hold any value in $[0,2000]$.

We use two methods to analyze effective size of variables. One is dynamic analysis, which runs programs and monitors the value of each variable. Dynamic analysis is one kind of simulation-based method whose results depend on input data sets given to the programs. The other is static analysis.

For static analysis, when the maximum value of an unsigned integer variable $x$ is $n_{\max }$, the effective size of $x$, $e(x)$, is given as follows:

$$
e(x)=\log _{2}\left(n_{\max }+1\right)
$$

For a signed integer $x$ with a maximum value $n_{\max }$ and a minimum value $n_{m i n}, e(x)$ is defined as follows:

$$
e(x)=\left\lceil\log _{2} \mathcal{N}\right\rceil+1
$$

where

$$
\mathcal{N}=\max \left(\left|n_{\max }\right|+1,\left|n_{\min }\right|\right)
$$

Static analysis is an efficient method to analyze the effective size of variables. However, in many cases when we can not predict the assigned value of a variable unless we execute the program, such as the case of unbounded loops, static analysis becomes insufficient. As a solution to this problem, we adopted dynamic analysis in our approach.

Figure3 shows a part of the algorithm used for dynamic analysis. In dynamic analysis, we execute the program $S$ with input data $D_{i n}$ and monitor the values $y_{i}$ assigned to each variable $n_{i}$. We insert the monitoring function to the assignment statement of variables. The arguments of the 


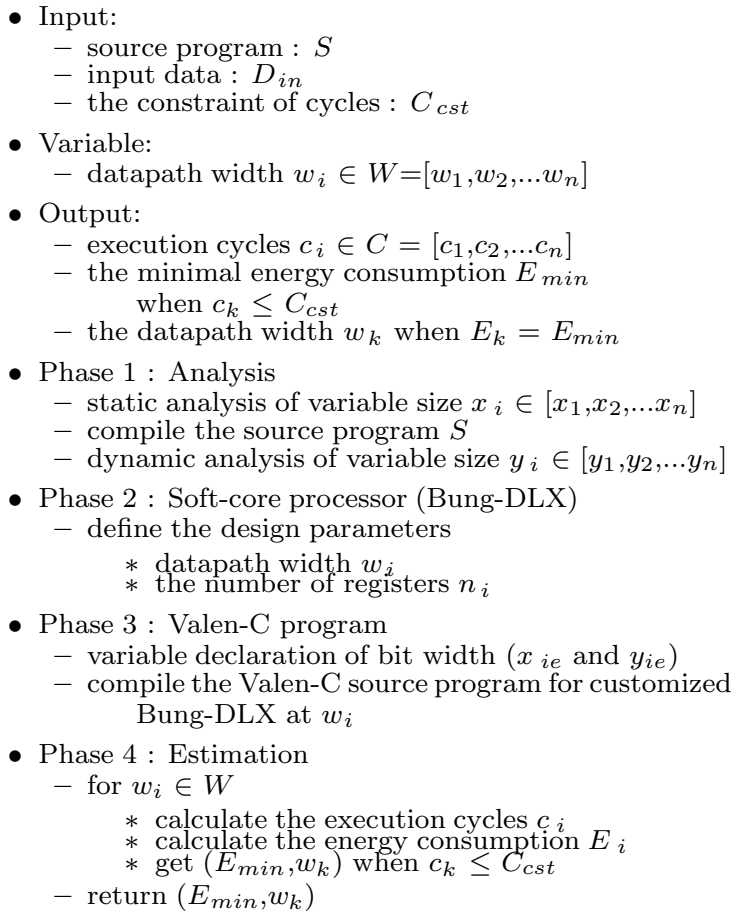

Figure 2: Pseudo code of the algorithm for energy minimization

monitoring function are the variable name $n_{i}$ and its assigned value $y_{i}$. The monitoring function checks the value assigned to the variable, verifies the bit width required and then memorize it. After that, it keeps the bit width temporarily in a table. When the monitoring function checks the same variable with a different assigned value, it compares the new bit width with the bit width already memorized in the table, and keeps the bigger one in the table and so on. Thus, the required bit width $y_{i e}$ of the variable $n_{i}$ is got after executing the program.

\subsection{Efficient Use of Data Memory}

Since in many cases, high-level specifications are devoted to describe functionalities of target systems rather than implementation details, they often contain a lot of redundancies such as duplicated computations and never executed code. Therefore, the specifications must be optimized to remove the redundancies for energy-efficient design. Some redundancies are introduced in size of variables. For example, in C programs, a variable whose value is between 0 and 1000 is often declared as the int type, i.e., usually 16 or 32 bits depending on target processors, and then some upper bits make nonsense. This means that the memory has many unnecessary bits, which do not essentially contribute to the calculation of programs. Therefore redundant bits should be removed to reduce power consumption.

C language provides for three integer sizes, declared using the keywords short, int and long. The compiler designer determines the sizes of these integer types. In many processors, the size of short is 16 bits, int is 16 or 32 bits, long is 32 bits. On the other hand, in Valen-C, programmers explicitly specify the required bit width of each integer data type. Thus it becomes possible to reduce the energy of the datapath and the data memory, which is dissipated by the redundant bits. For instance, if variables $\mathrm{x}, \mathrm{y}$, and $\mathrm{z}$ require

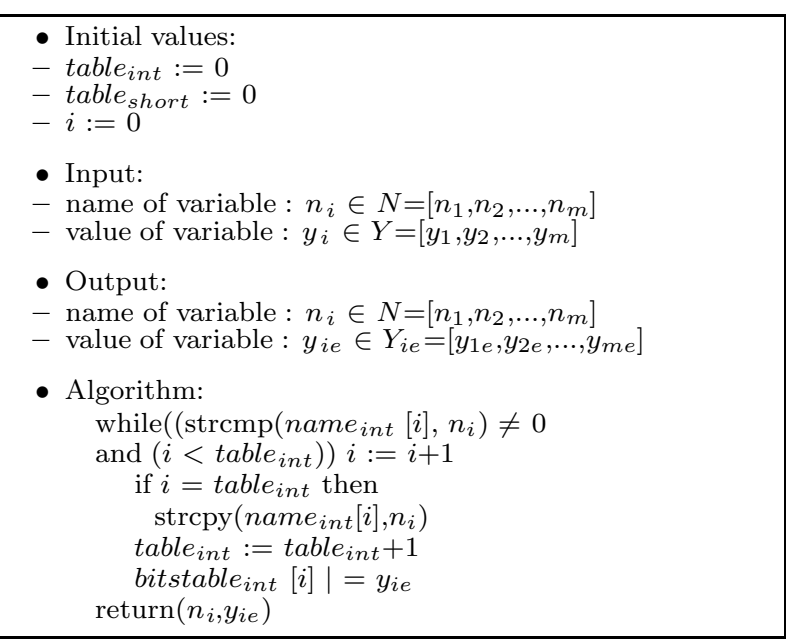

Figure 3: A part of the algorithm for dynamic analysis

12, 20 and 24 bits respectively, the programmer can write "int12 x; int20 y; int24 z;" in the variable declaration of Valen-C program. If a processor with a datapath width of 20 bits is used in the system, the total memory size will be 80 bits. Moreover, the unused bits in the data memory will be 24 bits. On the other hand, if a processor of a datapath width of 12 bits is used, the total data memory size will become only 60 bits, and the unused memory size will decrease to 4 bits. As a result, specifying the word length required for each variable and changing the datapath width have a significant role in reducing the data memory size of a system. Therefore it also affects the power consumption of the system.

\subsection{Datapath Width Optimization}

System designers can tune the value of the datapath width in accordance with the characteristics of target system to deliver most suited processor. Designers can reduce the datapath width until the single precision point (SPP) without performance loss[5]. SPP is the processor datapath width, which is equal to the bit width of the largest variable in a program. It is the smallest datapath width at which all instructions can remain single-precision. Designers may obtain better solutions, more power savings by shrinking the datapath less than SPP, under performance constraints. Figure4 shows the overview of our datapath width optimization algorithm.

\subsection{Power Versus Performance Tradeoff}

Minimizing power consumption is not simply an altruistic activity. A device consuming less power will accrue several desirable advantages such as longer battery life for wireless devices, but somewhat less obvious advantages, such as reliability and performance. The datapath width of a processor strongly affects the power consumption of the whole system including the processor, data memories and instruction memories, it also affects the execution cycles of a given task, i.e., narrowing the datapath width less than SPP will cause the increase of execution cycles because of multipleprecision operations. For example, that an addition of 20 bit data is executed by only one instruction on a 20 bit processor is assumed, If the datapath width becomes to 10 bits, two instructions including additions of lower 10 bits 


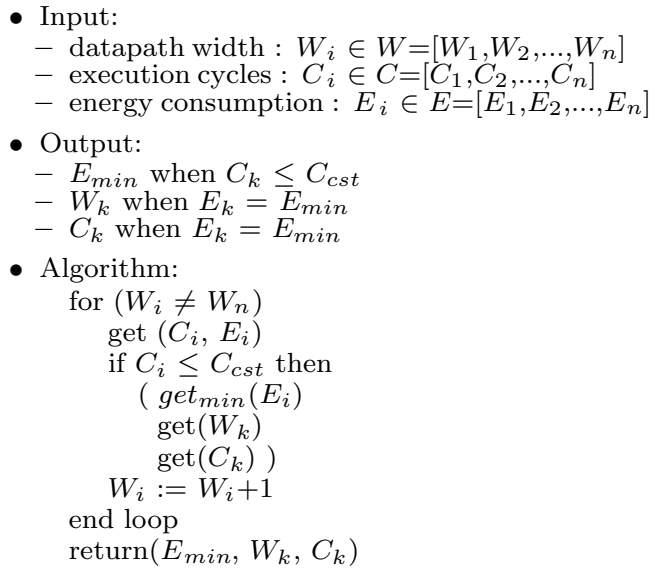

Figure 4: Pseudo code of the algorithm for datapath width optimization

and high 10 bits with carry are required. So trade-offs exist between datapath width and execution cycles. Although a processor with narrower datapath width dissipates lower power per clock cycle, the total energy for the task is not reduced always by narrowing the datapath width. Thus, for a given target system, trading off the power consumption and performance is an important work.

\section{ENERGY ESTIMATION MODELS}

This section describes energy consumption models. The total energy consumption, $E$, is the summation of energy consumed by the processor $\left(E_{\text {proc }}\right)$ and memories $\left(E_{m e m}\right)$.

$$
E=E_{\text {proc }}+E_{\text {mem }}
$$

We estimated $E_{\text {proc }}$ and $E_{m e m}$ separately, and got the energy consumption model of our soft-core processor generated by HITACH 0.5um CMOS technology and the energy consumption models of memory generated by Alliance CAD System Ver.3.0 with 0.5um double metal CMOS technology.

$E_{\text {proc }}$ is given by

$$
E_{\text {proc }}=\sum_{i \in I} e_{i} \times \text { Cycle }_{i}
$$

where

$e_{i}$ : Average energy of instruction $i$

Cycle $_{i}$ : The number of execution of instruction $i$

$I$ : Instruction set of Bung-DLX

$e_{i}$ is obtained by performing post-layout simulation of switch-level. After several simulations, we obtained the empirical energy model at several datapath widths in Figure 5 , where power savings are got by comparing to the power consumption of 32bits Bung-DLX. The power dissipation in static CMOS can be divided into static, dynamic and shortcircuit power. Because static power and short-circuit power are far less than dynamic power, we just focus on dynamic power, which consists of Cell Internal Power $\left(P_{c}\right)$ and Net Switching power $\left(P_{s}\right)$.

$e_{i}$ is shown as follows:

$$
e_{i}=\frac{1}{2} \times V_{d d}^{2} \sum_{n e t}\left[C(j) \times S(j)+E_{c(k)} \times S_{(k)}\right]
$$

where

$V_{d d}$ : Supply voltage

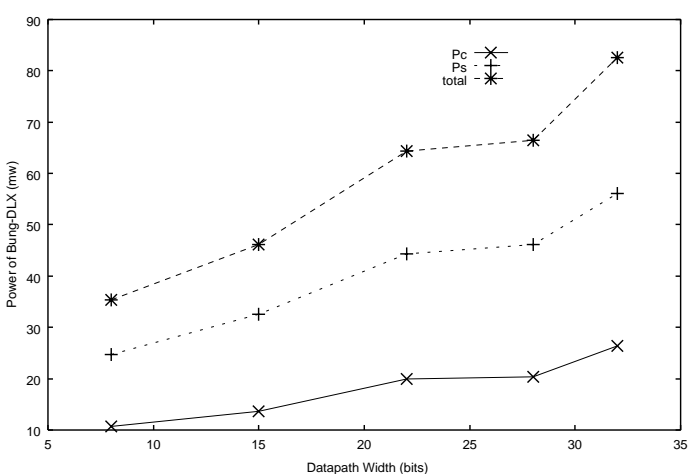

\begin{tabular}{|c|c|c|c|c|}
\hline $\begin{array}{c}\text { Datapath } \\
\text { Width(bit) }\end{array}$ & $\begin{array}{c}P_{c} \\
(\mathrm{mw})\end{array}$ & $\begin{array}{c}P_{s} \\
(\mathrm{mw})\end{array}$ & $\begin{array}{c}P_{\text {total }} \\
(\mathrm{mw})\end{array}$ & $\begin{array}{c}\text { Savings } \\
(\%)\end{array}$ \\
\hline \hline 32 & 26.39 & 56.15 & 82.54 & - \\
\hline 28 & 20.33 & 46.15 & 66.48 & 19.46 \\
\hline 22 & 19.95 & 44.39 & 64.34 & 22.05 \\
\hline 15 & 13.62 & 32.54 & 46.16 & 44.08 \\
\hline 8 & 10.67 & 24.69 & 35.36 & 57.16 \\
\hline
\end{tabular}

Figure 5: Power of Bung-DLX $\left(V_{d d}=3.3 \mathrm{~V}\right)$

$C(j)$ : Load capacitance of net $\mathrm{j}$

$S(j)$ : The average number of switching of net $\mathrm{j}$ per clock cycle

$E_{c(k)}$ : Internal power of cell $\mathrm{k}$

$S_{(k)}$ : The average number of switching of cell k per clock cycle

$E_{m e m}$ is estimated as follows:

$$
\begin{array}{r}
E_{\text {mem }}=E_{R O M}+E_{S R A M} \\
E_{R O M}=e_{R O M} \times \sum_{i \in I} \text { Cycle }_{i} \\
E_{S R A M}=e_{S r} \times \text { Cycle load }_{\text {lod }}+e_{S w} \times \text { Cycle }_{\text {store }}
\end{array}
$$

where

$e_{R O M}$ : Energy per read access to ROM

$e_{S r}\left(e_{S w}\right)$ : Energy per read (write) access to SRAM

Cycle load $\left(\right.$ Cycle $\left._{\text {store }}\right)$ : The number of read (write) accesses of SRAM

The access energy of memories $\left(e_{R O M}, e_{S r}, e_{S w}\right)$ is obtained from the SPICE simulation of several memories with the different configurations. As the result, we have obtained the estimation models as follows:

$$
\begin{aligned}
e_{R O M} & =50.97 * b * \sqrt{N_{\text {words }}}+1.4[\mathrm{pJ} / \text { cycle }] \\
e_{S r} & =24.9 * b * \sqrt{N_{\text {words }}}+56[\mathrm{pJ} / \text { cycle }] \\
e_{S w} & =197 * b * \sqrt{N_{\text {words }}}+369[\mathrm{pJ} / \text { cycle }]
\end{aligned}
$$

Where $b$ is the word width of the memory and $N_{w o r d s}$ is the number of words.

\section{EXPERIMENTS AND RESULTS}

In this section we present experiments and results based on several real applications to evaluate our proposed approach. We mainly illustrate how we use our approach to minimize energy consumption of MPEG-2 video decoder, a relatively large program.

In the experiments, we assumed the target system, a SOC chip, which consists a Bung-DLX processor, a ROM and a SRAM. Bung-DLX is a non-pipelined, simple RISC processor, which has several design parameters including the dat- 
Table 1: The number and types of the variables (MPEG-2 decoder)

\begin{tabular}{|c|c|c|c|}
\hline types & Num. & types & Num. \\
\hline int & 384 & unsigned & 35 \\
pointers & 101 & short & 6 \\
char & 3 & unsigned char & 21 \\
\hline
\end{tabular}

Table 2: Static analysis results(MPEG-2 decoder)

\begin{tabular}{|c|c|c|c|}
\hline E.Size & N.of Variables & E.Size & N. of Variables \\
\hline 1bit & 50 & 12 bits & 14 \\
2bits & 17 & 14 bits & 46 \\
3bits & 10 & 15 bits & 2 \\
4bits & 11 & 16 bits & 39 \\
5bits & 8 & 17 bits & 2 \\
6bits & 11 & 18 bits & 2 \\
7bits & 12 & 26 bits & 2 \\
8bits & 9 & 27 bits & 4 \\
9 bits & 7 & 28 bits & 3 \\
10bits & 3 & 29 bits & 3 \\
11bits & 6 & $30 \mathrm{bits}$ & 7 \\
\hline \hline Total & $5656 \mathrm{bits}$ & $-34 \%$ & $(8576 \mathrm{bits})$ \\
\hline
\end{tabular}

apath width and the number of registers. All instructions are executed within a single machine cycle. The ROM and the SRAM are used as instruction memory and data memory respectively. These memories are generated by Alliance CAD System Ver. 2.0 with $0.5 \mu m$ double metal CMOS technology. For simplicity, we assumed that no other core is integrated in the SOC chip.

\subsection{Variable Size Analysis for MPEG-2}

Our program is based on Mpeg2decode program from the MPEG Software Simulation Group. It is a player for MPEG1 and MPEG-2 video bitstreams. Mpeg2decode is an implementation of an ISO/IEC DIS 13818-2 decoder, whose emphasis is on correct implementation of the MPEG standard and comprehensive code structure. We rewrote it in Valen-C with about 6650 lines. The MPEG- 2 core consists of several function blocks such as a soft-core processor, IDCT blocks, a couple of motion estimation blocks, a motion compensation block, variable length encoding, decoding blocks and so on.

We analyzed the $\mathrm{C}$ source program of MPEG-2 video decoder and got some analysis results. The number and types of the variables in MPEG-2 decoder are described in Table 1. The results of static variable analysis are depicted in Table 2 (E.Size means effective size of variable; N.of Variables means the number of variables), and that of dynamic analysis are shown in Table 3 (V.name means variable name). From Table 2 and Table 3, we can see that there are many redundant bits in the variables of MPEG-2 decoder $\mathrm{C}$ source program. We got $34 \%$ reduction of bits from the static analysis and $52 \%$ from the dynamic analysis.

To verify our analysis results of variable size, we used the following model.

$$
P S N R=10 \times \log _{10}\left[\frac{1}{E} \times 255^{2}\right][d B]
$$

where, $P S N R$ : Ratio of pick signal to noise

$$
E \text { : Mean-square error }
$$

Our experimental results of $P S N R$ are infinite, so it shows that the variables, which are assumed according to the analysis results can work exactly as that of the source
Table 3: Dynamic analysis results(MPEG-2 decoder)

\begin{tabular}{|c|c|c|c|}
\hline V.name & E.size & V.name & E.size \\
\hline fn & 5 bits & g2nc & 7 bits \\
fl & $12 \mathrm{bits}$ & rbx & $12 \mathrm{bits}$ \\
sn & $6 \mathrm{bits}$ & rby & $12 \mathrm{bits}$ \\
nl & $20 \mathrm{bits}$ & rec4s1 & $24 \mathrm{bits}$ \\
gb32l & $20 \mathrm{bits}$ & rec4s4 & $24 \mathrm{bits}$ \\
gbl & $20 \mathrm{bits}$ & rec4cs1 & $24 \mathrm{bits}$ \\
gbn & $5 \mathrm{bits}$ & rec4cs4 & $24 \mathrm{bits}$ \\
g2ai & $20 \mathrm{bits}$ & rechs1 & $24 \mathrm{bits}$ \\
g2asign & $20 \mathrm{bits}$ & rechcs1 & $24 \mathrm{bits}$ \\
g2aincn & $18 \mathrm{bits}$ & rec4as1 & $24 \mathrm{bits}$ \\
g2anc & $6 \mathrm{bits}$ & rec4as4 & $24 \mathrm{bits}$ \\
gi & $7 \mathrm{bits}$ & rechas1 & $24 \mathrm{bits}$ \\
gsign & $20 \mathrm{bits}$ & rechas2 & $24 \mathrm{bits}$ \\
gincnt & $6 \mathrm{bits}$ & rec4acs1 & $24 \mathrm{bits}$ \\
g2i & $7 \mathrm{bits}$ & rec4acs2 & $24 \mathrm{bits}$ \\
g2sign & $3 \mathrm{bits}$ & rechacs1 & $24 \mathrm{bits}$ \\
g2incnt & $7 \mathrm{bits}$ & rechacs2 & $24 \mathrm{bits}$ \\
\hline \hline Toyal & $1056 \mathrm{bits}$ & $521 \mathrm{bits}$ & $-52 \%$ \\
\hline
\end{tabular}

program of MPEG-2 video decoder. Therefore, our analysis results are verified.

\subsection{Power and Performance Estimation}

This section reports some experimental data concerning the use of our approach to reduce energy consumption. The cycle count is obtained by using our instruction-level simulator. The input of the simulator is the assembly code, which is generated by the retargetable Valen-C compiler. Results of energy consumption $E_{t}$ (shown in Figure6) include energy of a soft-core processor $\left(E_{p}\right)$, a data RAM $\left(E_{s}\right)$ and an instruction $\operatorname{ROM}\left(E_{r}\right)$, where $D . W$ is datapath width. We use the energy consumption models in section 4.Apparently, the energy consumption changes nonlinearly.

Figure 7 shows the energy consumption, execution cycles and area (gates) of MPEG-2 video decoder, and we got the optimal datapath width, 28bits for MPEG-2 video decoder. Figure 8 describes the energy savings of our benchmarks, such as Lempel-Ziv algorithm, ADPCM encoder, and MPEG-2 AAC decoder and so on. No Opt. means the original datapath width of Bung-DLX (32bits). Opt. is the datapath width where the whole system has the minimization energy consumption without performance loss. For LempelZiv algorithm, we got energy savings of $48.3 \%$ at datapath width of $15 \mathrm{bits}$, for ADPCM encoder, energy savings is $22.8 \%$ at datapath width of 19 bits and for MPEG-2 video decoder, the energy savings is $10.8 \%$ at datapath width of 28 bits. For different application, the number of variables is different and the effective size of variables is also different, therefore the optimal datapath width of minimal energy is different. For a given application, our approach just tries to take advantage of the characteristics of the application to reduce the energy consumption.

\section{CONCLUSIONS}

In this paper, we have proposed a system-level energy minimization approach through datapath width optimization, which can suit the complexity of embedded systems and stringent time-to-market constraints. We also presented 


\begin{tabular}{|c|c|c|c|c|c|}
\hline \multirow{2}{*}{ D. W } & \multicolumn{5}{|c|}{ Supply Voltage $V_{d d}=3.3 V$} \\
\cline { 2 - 6 } & $E_{p}(J)$ & $E_{s}(J)$ & $E_{r}(J)$ & $E_{t}(J)$ & Savings \\
\hline \hline 32bits & 0.85 & 85.76 & 70.55 & 157.2 & - \\
\hline 30bits & 0.78 & 76.97 & 70.55 & 148.3 & $5.63 \%$ \\
\hline 28bits & 0.68 & 69.01 & 70.55 & 140.2 & $10.81 \%$ \\
\hline 26bits & 0.90 & 124.8 & 72.14 & 197.8 & $-25.8 \%$ \\
\hline 22bits & 1.03 & 108.6 & 109.8 & 219.4 & $-39.6 \%$ \\
\hline
\end{tabular}

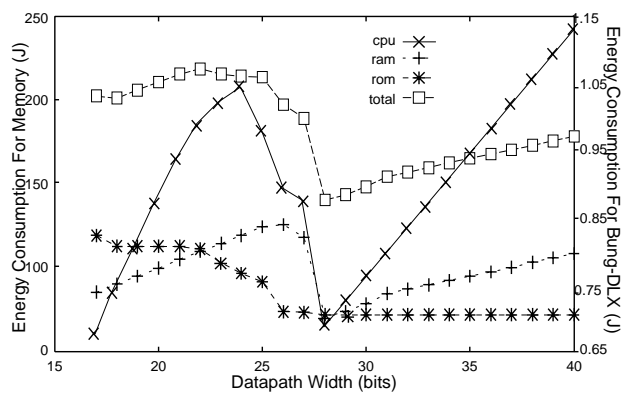

Figure 6: Energy consumption for MPEG-2 decoder

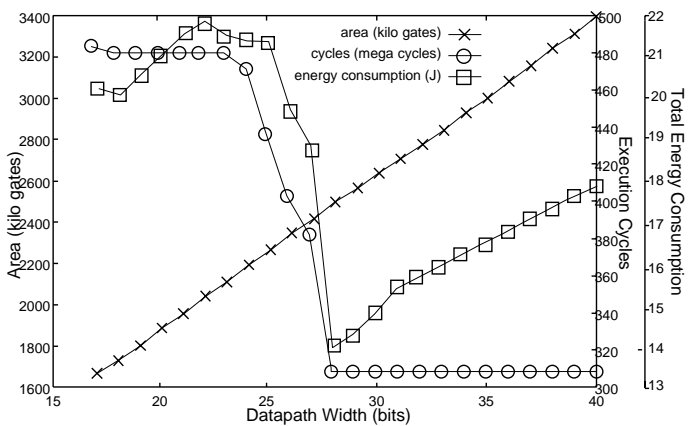

Figure 7: Energy consumption, execution cycles and area (gates) for MPEG-2 decoder

a set of algorithms that minimize energy consumption in system-level. We illustrated issues and tradeoffs involved in the design. Our experimental results show that for a given application we can reduce significantly the energy consumption by datapath width optimization. We have demonstrated energy savings without performance penalty range from about $10.8 \%$ to $48.3 \%$, which based on a number of real embedded applications. Extending parameter-tuning for low power to DSPs is our future work.

\section{ACKNOWLEDGMENTS}

This research was partly supported by the Grant-in Aid for Scientific Resarch (B) (2) 12558029 and VCDS project of STARC.

\section{REFERENCES}

[1] H.Yasuura, H.Tomiyama, A.Inoue, F.N.Eko, "Embedded System Design Using Soft-Core Processor and Valen-C", Journal of Information Science and Engineering, No.14, pp.587-603, August 1998

[2] A.Inoue, H.Tomiyama, T.Okuma, H.Kanbara and H.Yasuura, "Language and Compiler for Optimizing Datapath Width of Embedded Systems", IEICE Trans. Fundamentals, Vol. E81-A, No.12, pp. 2595-2604, Dec. 1998.

\begin{tabular}{|c|c|c||c|}
\hline \multirow{2}{*}{ Applications } & \multicolumn{3}{|c|}{ Energy Consumption(J) } \\
\cline { 2 - 4 } & No Opt. & Opt. & Savings \\
\hline \hline Lempel-Ziv & 0.9517 & 0.4919 & $48.3 \%$ \\
\hline ADPCM & 1.181 & 0.9187 & $22.8 \%$ \\
\hline MPEG2 & 157.16 & 140.24 & $10.8 \%$ \\
\hline
\end{tabular}

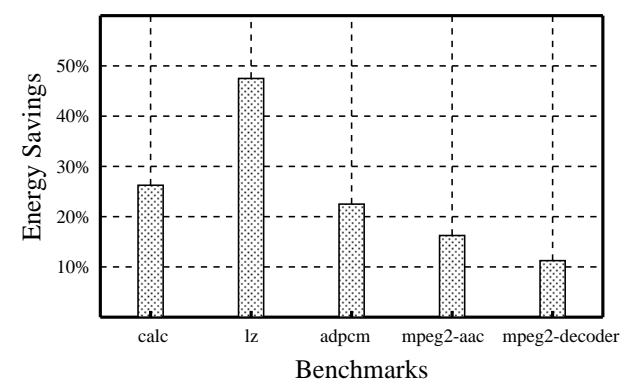

Figure 8: Energy savings for benchmarks

[3] F. N.Eko, A.Inoue, H.Tomiyama, H.Yasuura, "Soft-Core Processor Architecture for Embedded System Design", IEICE Trans.on Electronics, Vol.E81-C No.9, pp1416-1423, Sep.1998.

[4] E.N.Eko and H.Yasuura, "A Cycle-Accurate Simulator Toolkit for Soft-Core Processors", Proc.of Asia Pacific Conference on cHip Design Languages (APCHDL'99), pp.11-16, October 1999.

[5] B. Shackleford, M. Yasuda, E. Okushi, H. Koizumi, H.Tomiyama, H.Yasuura, "Embedded System Cost Optimization via Data Path Width Adjustment", IEICE Trans. Information and Systems, Vol.E80-D, No.10, pp974-981, October.1997.

[6] A.Inoue, T.Ishihara and H.Yasuura, "Flexible system lsi for embedded systems and its optimization techniques", Journal of Design Automation for Embedded System, 5(2), 2000.

[7] L.Benini, R.Hodgson and P.Siegel, "System-level Power Estimation And Optimization", International Symposium on Low Power Electronics and Design, pp.173-178, Aug.1998.

[8] P.Landman and J.Rabaey, "Architectural Power Analysis: The Dual Bit Type Method", IEEE Transactions on VLSI Systems, Vol.3, No.2, June 1995.

[9] T.Sato, M.Nagamatsu, H.Tago, "Power and Performance Simulator:ESP and its Application for 100 MIPS/W Class RISC Design", IEEE Proc.of Symposium on Low Power Electronics and Design, pp.46-47, 1994.

[10] I.Hong, D.Kirovski et al. , "Power Optimization of Variable voltage Core-Based Systems", IEEE Proc.of 35th. Design Automation Conference(DAC'98), pp.176-181,1998.

[11] U.Ko and P.Balsara, "Energy Optimization of Multilevel Cache Architectures for RISC and CISC Processors", IEEE Transactions on VLSI Systems, vol.6, no.2, pp.299-308, June 1998.

[12] Jorg Henkel, "A Low Power Hardware/software partitioning Approach for Core-Based Embedded Systems", IEEE Proc. of 36th. Design Automation Conference (DAC'99), pp.122-127, 1999. 